Nonlinear Mathematical Physics 1997, V.4, N 1-2, 180-189.

\title{
Nonlinear Quantum Dynamical Equation for Self-Acting Electron
}

\author{
V.P. OLEINIK \\ Department of General and Theoretical Physics, 32-60, \\ Kyiv Polytechnic Institute, 37 Peremogy Avenue, Kyïv, 252056, Ukraïna \\ Fax: (044) 274-59-32
}

\begin{abstract}
From the action principle, the quantum dynamical equation is obtained both relativistically and gauge invariant, which is analogous to the Dirac equation and describes behaviour of an arbitrary number of self-acting charged particles. It is noted that solutions of this equation are indicative of the soliton nature of an electron and allow to determine the internal energy, dimensions and geometric shape of the electron in different quantum states. The theory proposed represents a synthesis of the standard QED and ideas of the self-organization theory of physical systems.
\end{abstract}

\section{Introduction}

Development of an uncontradictory quantum model of an electron which would take into account Coulomb self-action of the particle remains one of key problems of present-day physics. Numerous attempts to work out such a model have failed. In our opinion, the main reason consists in using the standard scheme of quantum mechanics whose framework proved to be too narrow to describe the electron's self-action in a self-consistent way.

One of the most bold ideas concerning physical nature of the electron belongs to Schrödinger who believed that dimensions of the electron are the same as those of the atom [1]. According to Schrödinger's interpretation of quantum mechanics, the quantity $e|\psi|^{2}$ is the density of spatial distribution of electron's charge ( $e$ and $\psi$ are a charge and wave function of the particle, respectively).

An important stride towards elucidating the true physical nature of the electron was made by Barut and his collaborators [2-5]. They managed to formulate and develop quantum electrodynamics (QED) based entirely on the self-energy picture (the Self-Field QED) without treating the second quantization of electromagnetic field. As was pointed out in [3], the correct equation of motion for the radiating electron is not the Dirac or Schrödinger equation for a bare electron, but an equation with an additional nonlinear self-energy term. This viewpoint is reciprocated by many investigators, among which are Fushchych and his collaborators who have been deeply involved in the search for the nonlinear equation for the electron $[6,7]$.

In [8-11] new lines of the approach to the problem are proposed which change QED into a theory of self-organization of the electrically charged matter. A mechanism of selforganization consists in the back action of the Coulomb field created by the particle upon the same particle and is described by the model of an open system with the wave functions belonging to the indefinite metric space.

Copyright (c) 1997 by Mathematical Ukraina Publisher. All rights of reproduction in any form reserved. 
Basic to the approach are the following physical ideas:

1. The electron is a quantum (an elementary excitation) of the charged matter field localized in a bounded region of space and subject to Coulomb self-action. This means that the abilities of the electron to produce Coulomb forces and Coulomb self-action are the physical properties intrinsically inherent in the charged matter and should be included from the very beginning in the definition of the particle. Mathematically, from this it follows that the behaviour of the electron should be governed by the nonlinear dynamical equation. Physically, the electron becomes a self-organizing system, whose geometric shape and linear dimensions are determined in a self-consistent way from the solutions of a dynamical equation.

2. Since the electron is a clot of the charged matter producing the long-range Coulomb forces in the surroundings, its environment becomes a medium which can have a determining effect on the physical properties of the particle. Thus, the electron turns to an open system inseparable from the surrounding medium. In this sense, the whole universe takes part in the formation of the electron.

The aim of this article is to briefly outline the main results of the theory of a self-organizing electron which represents the synthesis of the standard QED and theory of self-organization in physical systems [12]. The foundation of the theory is the relativistically invariant action which takes into account both the Coulomb self-action and interaction of charged particles the transverse electromagnetic waves and is based on the model of open system. The fundamental dynamical equation derived from the action principle $[8,11]$ is a generalization of the Dirac equation to the case of the self-organizing electron. The solutions to this equation are indicative of the soliton nature of the electron and allow one to determine the internal energy, dimensions and geometric shape of the particle in different quantum states. It should be emphasized that the theory proposed fits the fundamental principles of symmetry, gives insight into the problem of electron's stability and does not lead to divergence of the self-energy. The calculations of hydrogen atom dimensions, Balmer spectrum and total angular momentum of the electron, which have been made to date, are in agreement with experimental data.

Section 2 deals with the equation of motion for the self-acting electron in the nonrelativistic approximation. In Section 3 the relativistic generalization of the fundamental dynamical equation is obtained. The main results of the paper are summarized in Conclusion.

\section{Nonrelativistic equation for electron}

In order to represent the Coulomb field produced by the electron as one of its physical properties, we need to derive the dynamical equation which allows for the Coulomb selfaction. One of the hints as to how to do this can be obtained from Maxwell's equations for electric and magnetic fields. From them it follows that the total energy $W$ of the Coulomb field can be written as

$$
W=\frac{1}{2} \int \mathrm{d} \vec{r}_{1} \int \mathrm{d} \vec{r}_{2}\left|\vec{r}_{1}-\vec{r}_{2}\right|^{-1} \rho\left(\vec{r}_{1}, t\right) \rho\left(\vec{r}_{2}, t\right),
$$


$\rho=\rho(\vec{r}, t)$ being the charge density of the particles. Quantity (1) is the potential energy of the Coulomb interaction between the charges including energy of each particle. Obviously, when deriving the equation of motion for the self-acting electron from the action principle, we have to include the additional term $-W$ in the Lagrangian $L$ of the electron field, i.e.,

$$
L=L_{0}-W
$$

where $L_{0}$ is a Lagrangian for a free particle in the absence of the Coulomb field. Making use of the nonrelativistic approximation,

$$
L_{0}=\int \mathrm{d} \vec{r}\left(\frac{i}{2} \Psi^{*} \stackrel{\leftrightarrow}{\partial_{t}} \Psi-\frac{1}{2 m}\left(\vec{\nabla} \Psi^{*}\right)(\vec{\nabla} \Psi)\right)
$$

and putting

$$
\rho=e \Psi^{*} \Psi
$$

we arrive from the action principle at the following equation for the wave function $\Psi=$ $\Psi(\vec{r}, t)$ of the nonrelativistic electron:

$$
\begin{aligned}
i \frac{\partial \Psi}{\partial t} & =\left(-\frac{1}{2 m} \vec{\nabla}^{2}+U\right) \Psi \\
U(\vec{r}, t) & =e^{2} \int \mathrm{d} \vec{r}_{1}\left|\vec{r}-\vec{r}_{1}\right|^{-1}\left|\Psi\left(\vec{r}_{1}, t\right)\right|^{2} \equiv U .
\end{aligned}
$$

An inspection shows, however, that equation (5) with the potential energy function (6) has no solutions satisfying reasonable physical requirements. From the physical point of view, this is due to the fact that Coulomb forces of repulsion are trying to tear the electron to pieces. Formally, the potential energy function $U(6)$ is a potential hump rather than a potential well and so equation (5) cannot have solutions that would describe stable states of the particle.

Thus, a negative result is obtained: we had a try, remaining within the framework of the standard theoretical scheme, to take into account self-action of the electron and arrived at the equation that has no reasonable physical solutions at all. This result seems to mean that it is impossible to construct, without resorting to essentially new physical ideas, a consistent quantum model of the electron.

As was noted in Introduction, the self-acting electron differs essentially from a bare electron in its physical properties. The main difference is that the real electron, as distinct from the bare one, produces the long-range Coulomb field in the surroundings and as a result, cannot be treated as an isolated system.

To take into account that the real electron is inseparably linked with the surrounding medium, we should first of all increase the number of dynamical variables describing it. Here we adopt the simplest version of the theory in which the number of variables is doubled as compared with the isolated system, namely, to each dynamical variable of the bare electron, $\Psi$, there correspond two dynamical variables which are denoted by $\Psi$ and $\widetilde{\Psi}$. These quantities are considered as components of the wave function describing the quantum state of particle. One of them, say, $\Psi$, corresponds in a sense to the particle alone (to the bare electron) and the other, $\widetilde{\Psi}$, to the surrounding medium in which the particle moves. 
The fundamental quadratic form defining the metric of the wave function space is assumed to be given by

$$
\widetilde{\Psi}^{*} \Psi+\Psi^{*} \widetilde{\Psi} .
$$

This quantity is used instead of the positively defined quadratic form $\Psi^{*} \Psi$ underlying the conventional formulation of quantum mechanics. As the electric charge density we take the quantity

$$
\rho(x)=e\left(\widetilde{\Psi}^{*}(x) \Psi(x)+\Psi^{*}(x) \widetilde{\Psi}(x)\right), \quad x=(t, \vec{r})
$$

and as the Lagrangian of the free electron field we use the function (cf (3))

$$
L_{0}=\int \mathrm{d} \vec{r}\left\{\frac{i}{2}\left(\widetilde{\Psi}^{*} \stackrel{\leftrightarrow}{\partial_{t}} \Psi+\Psi^{*} \stackrel{\leftrightarrow}{\partial_{t}} \widetilde{\Psi}\right)-\frac{1}{2 m}\left[\left(\vec{\nabla} \widetilde{\Psi}^{*}\right)(\vec{\nabla} \Psi)+\left(\vec{\nabla} \Psi^{*}\right)(\vec{\nabla} \widetilde{\Psi})\right]\right\} .
$$

The Lagrangian $L$ of the nonrelativistic self-acting electron is given by (2) where $W$ and $\rho$ are defined by (1) and (8), respectively. The action principle with the Lagrangian $L(2)$ gives rise to the following nonlinear equations of motion

$$
\begin{array}{r}
\left(i \frac{\partial}{\partial t}+\frac{\vec{\nabla}^{2}}{2 m}-U(x)\right)\left(\begin{array}{c}
\Psi(x) \\
\widetilde{\Psi}(x)
\end{array}\right)=0 ; \\
U(x)=e \int \mathrm{d} \vec{r}_{1}\left|\vec{r}-\vec{r}_{1}\right|^{-1} \rho\left(\vec{r}_{1}, t\right),
\end{array}
$$

the quantity $\rho(\vec{r}, t)$ being given by (8).

Analysis shows that equations (10) have solutions describing the stationary states of the electron at $N=-1$ where $N$ is the normalization constant

$$
N=\int \mathrm{d} \vec{r}\left(\widetilde{\Psi}^{*} \Psi+\Psi^{*} \widetilde{\Psi}\right) .
$$

As is seen from (7), the wave functions of the self-acting electron belong to the indefinite metric space. The presence of two components of the nonrelativistic electron wave function without regard for the spin variables, $\Psi$ and $\widetilde{\Psi}$, implies that the particle has an additional degree of freedom. In the theory under consideration this degree of freedom is characterized by the sign of the normalizing factor $N(N= \pm 1)$ which acts as a quantum number taking into account the Coulomb self-action of the particle. It is of interest that the states of the free electron and atomic one differ from one another by the sign of $N: N=+1$ for the atomic electron and $N=-1$ for the free one [10, 13].

In connection with the quadratic form (7) it should be stressed that there is no way of describing the electron as an open system without using the indefinite metric space. This point is worthy of special attention because the quadratic form determines the properties of the wave function space and thus the physical behaviour of the system. At first sight, the quadratic form corresponding to the functional space of two variables, $\Psi$ and $\widetilde{\Psi}$, should be given by

$$
\Psi^{*} \Psi+\widetilde{\Psi}^{*} \widetilde{\Psi}
$$

instead of (7). Accordingly, the Lagrangian $\widetilde{L}=\widetilde{L}(\Psi, \widetilde{\Psi})$ of the real electron should be constructed in the standard manner:

$$
\widetilde{L}(\Psi, \widetilde{\Psi})=L_{1}(\Psi)+L_{2}(\widetilde{\Psi})+L_{\text {int }}(\Psi, \widetilde{\Psi})
$$


where $L_{1}(\Psi)$ is the Lagrangian of the bare electron ( that is, of the electron isolated from the medium), $L_{2}(\widetilde{\Psi})$ is the Lagrangian of the medium created by the particle and estranged from it, and $L_{\text {int }}$ the Lagrangian describing the interaction of the bare electron with the medium, with the equalities

$$
L_{\text {int }}(0, \widetilde{\Psi})=L_{\text {int }}(\Psi, 0)=0
$$

being fulfilled. If we now neglect the dynamical variables of the medium, that is, if we put $\widetilde{\Psi}=0$, we shall come to the Lagrangian of the bare electron

$$
\widetilde{L}(\Psi, 0)=L_{1}(\Psi) \neq 0
$$

being considered as zeroth approximation for the real particle. The other limiting case, $\Psi=0$, leads to the Lagrangian of the medium alone

$$
\widetilde{L}(0, \widetilde{\Psi})=L_{2}(\widetilde{\Psi}) \neq 0 .
$$

We should take into account, however, that the real electron is indissolubly related to the surrounding medium. The two objects, the bare electron and the bare medium created by it, do not exist in nature separately. Therefore, the use of the Lagrangian (14) under the conditions (15) and (16), as a basis of the theory is intolerable. At the same time, using the quadratic form (7) and accordingly the Lagrangian $L=L(\Psi, \widetilde{\Psi})(2)$, where $L_{0}, W$ and $\rho$ are defined by (9), (1) and (8), we allow for inseparability of the particle from the medium. In particular, the equalities

$$
L(\Psi, 0)=L(0, \widetilde{\Psi})=0
$$

are fulfilled which mean that the bare electron approximation has no physical meaning; in either case, with no electron or with no medium, we have no physical system.

\section{Relativistic generalization}

Let's introduce some designations which are necessary for deriving the relativistic fundamental equation of motion. As is known, any vector field, say, $\vec{E}=\vec{E}(\vec{r})$, can be split into potential $\left(\vec{E}_{\|}\right)$and vortex $\left(\vec{E}_{\perp}\right)$ components, $\vec{E}=\vec{E}_{\|}+\vec{E}_{\perp}$, which are defined by

$$
\begin{aligned}
& \vec{\nabla} \times \vec{E}_{\|}=0 ; \quad \vec{\nabla} \vec{E}_{\|} \neq 0 \quad(\text { or }=0), \\
& \vec{\nabla} \times \vec{E}_{\perp} \neq 0 ; \quad \vec{\nabla} \vec{E}_{\perp}=0 .
\end{aligned}
$$

Analogously, any 4 -vector field $A^{\mu}=\left(A^{0}, \vec{A}\right)$ can be represented as a sum of potential $\left(A_{\|}^{\mu}\right)$ and vortex $\left(A_{\perp}^{\mu}\right)$ components, $A^{\mu}=A_{\|}^{\mu}+A_{\perp}^{\mu}$, with

$$
A_{\|}^{\mu}=\left(A^{0}, \vec{A}_{\|}\right), \quad A_{\perp}^{\mu}=\left(0, \vec{A}_{\perp}\right) .
$$

Splitting the vector fields entering into Maxwell's equations for the electric $(\vec{E})$ and magnetic $(\vec{B})$ fields into the vortex and potential components, we arrive at the two independent subsets of equations:

$$
\begin{array}{llrl}
\partial_{t} \vec{B} & =-\vec{\nabla} \times \vec{E}_{\perp}, & & \vec{\nabla} \vec{B}=0, \\
\partial_{t} \vec{E}_{\perp}=\vec{\nabla} \times \vec{B}-4 \pi \vec{j}_{\perp}, & & \vec{\nabla} \vec{E}_{\perp}=0
\end{array}
$$


and

$$
\vec{\nabla} \vec{E}_{\|}=4 \pi \rho, \quad \partial_{t} \vec{E}_{\|}=-4 \pi \vec{j}_{\|}, \quad \vec{\nabla} \times \vec{E}_{\|}=0 .
$$

Each subset is seen from (19) and (20) to involve merely either the vortex components or the potential ones. These subsets can also be represented in the 4-vector form:

$$
\partial_{\nu} \mathcal{F}_{A}^{\mu \nu}=-4 \pi j_{A}^{\mu} ; \quad \partial^{\alpha} \mathcal{F}_{A}^{\mu \nu}+\partial^{\mu} \mathcal{F}_{A}^{\nu \alpha}+\partial^{\nu} \mathcal{F}_{A}^{\alpha \mu}=0,(A=\|, \perp)
$$

where $\partial_{\nu}=\frac{\partial}{\partial x^{\nu}}=\left(\partial_{t}, \vec{\nabla}\right) ; \quad x^{\nu}=(t, \vec{r}),(\nu=0,1,2,3) ; \quad \mathcal{F}_{\perp}^{\mu \nu}$ and $\mathcal{F}_{\|}^{\mu \nu}$ are the vortex and potential components of the field-strength tensor $\mathcal{F}^{\mu \nu}$ defined by

$$
\mathcal{F}_{\perp}^{\mu \nu}=\left(\begin{array}{cccr}
0 & -E_{\perp x} & -E_{\perp y} & -E_{\perp z} \\
E_{\perp x} & 0 & -B_{z} & B_{y} \\
E_{\perp y} & B_{z} & 0 & -B_{x} \\
E_{\perp z} & -B_{y} & B_{x} & 0
\end{array}\right) ; \mathcal{F}_{\|}^{\mu \nu}=\left(\begin{array}{cccc}
0 & -E_{\| x} & -E_{\| y} & -E_{\| z} \\
E_{\| x} & 0 & 0 & 0 \\
E_{\| y} & 0 & 0 & 0 \\
E_{\| z} & 0 & 0 & 0
\end{array}\right)
$$

$j_{\perp}^{\mu}(x)$ and $j_{\|}^{\mu}(x)$ are the components of the 4-current density $j^{\mu}(x)=(\rho(x), \vec{j}(x))$. It should be pointed out that the vortex $\left(A_{\perp}^{\mu}\right)$ and potential $\left(A_{\|}^{\mu}\right)$ components of the 4-vector $A^{\mu}$ each taken separately are not 4 -vectors. Analogously, the quantities $\mathcal{F}_{\perp}^{\mu \nu}$ and $\mathcal{F}_{\|}^{\mu \nu}$ do not behave like 4-tensors. Nevertheless, one can easily be convinced of form-invariance of Maxwell's equations (21) under Lorentz transformations.

Since the potential component of the electric field, $\vec{E}_{\|}$, is not an independent degree of freedom of the electromagnetic field, we include it in the definition of the elecrically charged matter to obtain the self-acting field. The vortex electromagnetic field will be treated on the same grounds as the charged matter field, using the indefinite metric space. To each dynamical variable we shall put into correspondence two variables; for one of them the old designation will be retained $\left(\vec{E}_{\perp}, \vec{B}\right.$ or $\left.\mathcal{F}_{\perp}^{\mu \nu}\right)$ and the other will be labelled by the sign "widetilde" $\left(\overrightarrow{\widetilde{E}}_{\perp}, \overrightarrow{\widetilde{B}}\right.$ or $\left.\widetilde{\mathcal{F}}_{\perp}^{\mu \nu}\right)$. Maxwell's equations for the additional variables are supposed to have the usual form analogous to (21).

The action of the whole system which consists of $n$ electrically charged fields described by $\Psi_{k}$ and $\widetilde{\Psi}_{k}(k=1,2, \ldots, n)$ and of the vortex electromagnetic fields represented by $\mathcal{F}_{\perp}^{\mu \nu}$ and $\widetilde{\mathcal{F}}_{\perp}^{\mu \nu}$ can be written as

$$
\begin{aligned}
S= & S_{\|}+S_{\perp}+S_{\text {int }} ; \\
S_{\|}= & \sum_{k=1}^{n} \int d^{4} x\left(\overline{\widetilde{\Psi}}_{k}\left(\frac{i}{2} \overleftrightarrow{\hat{\partial}}-m_{k}\right) \Psi_{k}+\bar{\Psi}_{k}\left(\frac{i}{2} \overleftrightarrow{\hat{\partial}}-m_{k}\right) \widetilde{\Psi}_{k}\right)- \\
& \frac{1}{2} \int d^{4} x_{1} \int d^{4} x_{2} \delta\left(\left(x_{1}-x_{2}\right)^{2}\right) j_{\| \mu}\left(x_{1}\right) j_{\|}^{\mu}\left(x_{2}\right) ; \\
S_{\perp}= & -\frac{1}{16 \pi} \int d^{4} x \widetilde{\mathcal{F}}_{\perp}^{\mu \nu}(x) \mathcal{F}_{\perp \mu \nu}(x) ; \\
S_{\text {int }}= & -\int d^{4} x j_{\perp \mu}(x) A_{\perp}^{\mu}(x),
\end{aligned}
$$

where $S_{\|}$is the action of the self-acting charged fields, $\Psi_{k}$ and $\widetilde{\Psi}_{k}$ are the wave function components for a particle of mass $m_{k}$ and electric charge $e_{k}(k=1,2, \ldots, n) ; S_{\perp}$ is the action of the vortex electromagnetic fields; $S_{\text {int }}$ describes interaction of charged particles 
with the electromagnetic fields; $j_{\|}^{\mu}$ and $j_{\perp}^{\mu}$ are the potential and vortex components of the 4-current density

$$
\begin{aligned}
j^{\mu}(x) & =\sum_{k=1}^{n} e_{k}\left(\overline{\widetilde{\Psi}}_{k}(x) \gamma^{\mu} \Psi_{k}(x)+\bar{\Psi}_{k}(x) \gamma^{\mu} \widetilde{\Psi}_{k}(x)\right) \\
& =(\rho(x), \vec{j}(x))
\end{aligned}
$$

$\widetilde{\mathcal{F}}_{\perp}^{\mu \nu}$ and $\mathcal{F}_{\perp}^{\mu \nu}$ are vortex components of the field-strength tensors $\widetilde{\mathcal{F}}^{\mu \nu}(x)$ and $\mathcal{F}^{\mu \nu}(x) ;$

$$
\begin{aligned}
\widetilde{\mathcal{F}}^{\mu \nu}(x) & =\partial^{\mu} \widetilde{\mathcal{A}}^{\nu}(x)-\partial^{\nu} \widetilde{\mathcal{A}}^{\mu}(x) ; \quad \widetilde{\mathcal{A}}=\widetilde{\mathcal{A}}_{\perp}+\widetilde{\mathcal{A}}_{\|} ; \\
\mathcal{F}^{\mu \nu}(x) & =\partial^{\mu} \mathcal{A}^{\nu}(x)-\partial^{\nu} \mathcal{A}^{\mu}(x) ; \quad \mathcal{A}=\mathcal{A}_{\perp}+\mathcal{A}_{\|} ; \\
\mathcal{A}_{\|}^{\mu}(x) & =\widetilde{\mathcal{A}}_{\|}^{\mu}(x)=\int d^{4} x_{1} \delta\left(\left(x-x_{1}\right)^{2}\right) j_{\|}^{\mu}\left(x_{1}\right) ; \\
\mathcal{A}_{\perp}^{\mu}(x) & =-\frac{1}{4 \pi} \int d^{4} x_{1} \delta\left(\left(x-x_{1}\right)^{2}\right) \partial_{1 \nu} \mathcal{F}_{\perp}^{\mu \nu}\left(x_{1}\right) ; \\
\widetilde{\mathcal{A}}_{\perp}^{\mu}(x) & =-\frac{1}{4 \pi} \int d^{4} x_{1} \delta\left(\left(x-x_{1}\right)^{2}\right) \partial_{1 \nu} \widetilde{\mathcal{F}}_{\perp}^{\mu \nu}\left(x_{1}\right) ; \\
A^{\mu}(x) & =\frac{1}{2}\left(\mathcal{A}^{\mu}(x)+\widetilde{\mathcal{A}}^{\mu}(x)\right) ;
\end{aligned}
$$

$\hat{\partial}=\partial_{\alpha} \gamma^{\alpha}, \gamma^{\alpha}$ are Dirac's matrices.

The action principle $\delta S=0$ gives rise to the fundamental dynamical equations for the charged particles $(k=1,2, \ldots, n)$

$$
\left(i \hat{\partial}-e_{k} \hat{A}(x)-m_{k}\right)\left(\begin{array}{c}
\Psi_{k}(x) \\
\widetilde{\Psi}_{k}(x)
\end{array}\right)=0
$$

and to Maxwell's equations

$$
\begin{aligned}
& \partial_{\nu} \widetilde{\mathcal{F}}_{\perp}^{\mu \nu}=\partial_{\nu} \mathcal{F}_{\perp}^{\mu \nu}=-4 \pi j_{\perp}^{\mu}, \\
& \partial^{\alpha} \widetilde{\mathcal{F}}_{\perp}^{\mu \nu}+\partial^{\mu} \widetilde{\mathcal{F}}_{\perp}^{\nu \alpha}+\partial^{\nu} \widetilde{\mathcal{F}}_{\perp}^{\alpha \mu}=\partial^{\alpha} \mathcal{F}_{\perp}^{\mu \nu}+\partial^{\mu} \mathcal{F}_{\perp}^{\nu \alpha}+\partial^{\nu} \mathcal{F}_{\perp}^{\alpha \mu}=0 .
\end{aligned}
$$

By their appearance the equations of motion (26) coincide with the Dirac equation for a charged particle in an external field described by a 4-potential $A^{\mu}=A_{\|}^{\mu}+A_{\perp}^{\mu}$. However, in distinction to Dirac's equation, they are nonlinear and nonlocal, with the nonlocality being of the space and time character. The quantities $A_{\|}^{\mu}$ and $A_{\perp}^{\mu}$ entering into (26) differ from each other by their physical nature: the first describes the Coulomb field and is expressed in terms of the wave functions of particles, and the second describes the vortex electromagnetic fields and is uniquely determined by the field variables $\vec{E}_{\perp}, \overrightarrow{\widetilde{E}}_{\perp}, \vec{B}, \overrightarrow{\widetilde{B}}$. These two considerably different quantities are combined in our theory to form a single 4-vector.

Excluding the vortex components $\widetilde{\mathcal{F}}_{\perp}^{\mu \nu}$ and $\mathcal{F}_{\perp}^{\mu \nu}$ of the field-strength tensors from the general expression (23) for the action with the help of Maxwell's equations (27), we arrive at

$$
\begin{aligned}
\widetilde{S}= & \sum_{k=1}^{n} \int d^{4} x\left[\overline{\widetilde{\Psi}}_{k}\left(\frac{i}{2} \stackrel{\hat{\partial}}{2}-m_{k}\right) \Psi_{k}+\bar{\Psi}_{k}\left(\frac{i}{2} \stackrel{\hat{\partial}}{2}-m_{k}\right) \widetilde{\Psi}_{k}\right]- \\
& \frac{1}{2} \int d^{4} x_{1} \int d^{4} x_{2} \delta\left(\left(x_{1}-x_{2}\right)^{2}\right) j_{\alpha}\left(x_{1}\right) j^{\alpha}\left(x_{2}\right) .
\end{aligned}
$$


If we put $\widetilde{\Psi}_{k}=\Psi_{k}$ in $(24)$ and (28), we shall come to the Self-Field QED by Barut [2-5].

The action principle $\delta \widetilde{S}=0$ gives the following equations of motion:

$$
\begin{aligned}
& \left(i \hat{\partial}-e_{k} \hat{A}(x)-m_{k}\right)\left(\begin{array}{c}
\Psi_{k}(x) \\
\widetilde{\Psi}_{k}(x)
\end{array}\right)=0, \\
& A^{\mu}(x)=\int d^{4} x_{1} \delta\left(\left(x-x_{1}\right)^{2}\right) j^{\mu}\left(x_{1}\right) .
\end{aligned}
$$

Using expression (28) one can derive in the usual manner the energy-momentum tensor $T^{\mu \nu}$ for the system of $n$ self-acting charged fields $\left(g_{\mu \nu}=0\right.$ at $\mu \neq \nu, \quad g_{00}=-g_{i i}=1, i=$ $1,2,3)$

$$
T^{\mu \nu}=t^{\mu \nu}+\theta^{\mu \nu}+\frac{1}{2} g^{\mu \nu} j_{\alpha} A^{\alpha}-A^{\mu} j^{\nu}
$$

where

$$
\begin{aligned}
t^{\mu \nu}= & \frac{i}{2} \sum_{k=1}^{n}\left(\overline{\widetilde{\Psi}}_{k} \stackrel{\leftrightarrow}{\partial^{\mu}} \gamma^{\nu} \Psi_{k}+\bar{\Psi}_{k} \stackrel{\leftrightarrow}{\partial^{\mu}} \gamma^{\nu} \widetilde{\Psi}_{k}\right)- \\
& g^{\mu \nu}\left\{\sum_{k=1}^{n}\left[\overline{\widetilde{\Psi}}_{k}\left(\frac{i}{2} \stackrel{\leftrightarrow}{\hat{\partial}}-m_{k}\right) \Psi_{k}+\bar{\Psi}_{k}\left(\frac{i}{2} \stackrel{\leftrightarrow}{\hat{\partial}}-m_{k}\right) \widetilde{\Psi}_{k}\right]-\frac{1}{2} j_{\alpha} A^{\alpha}\right\}, \\
\theta^{\mu \nu}= & -\frac{1}{4 \pi}\left(F^{\mu \alpha} F_{\alpha}^{\nu}-\frac{1}{4} g^{\mu \nu} F^{\alpha \beta} F_{\alpha \beta}\right) .
\end{aligned}
$$

The components of the 4-tensor $F^{\mu \nu}=\partial^{\mu} A^{\nu}-\partial^{\nu} A^{\mu}$ satisfying the equality

$$
\partial_{\nu} F^{\mu \nu}=-4 \pi j^{\mu}
$$

can be represented in the form $F^{\mu \nu}=F_{\|}^{\mu \nu}+F_{\perp}^{\mu \nu}$, with $F_{\|}^{\mu \nu}$ describing the Coulomb field and $F_{\perp}^{\mu \nu}$ the vortex fields. It should be stressed that in the model under study the field described by $F^{\mu \nu}$ is not a degree of freedom independent of charged particles. For this reason the equalities (32) are identities, not equations. The 4-tensor $\theta^{\mu \nu}$ (31) is an energy-momentum tensor for the field produced by the charged matter. It satisfies the equation

$$
\partial_{\nu} \theta^{\mu \nu}=-F^{\mu}
$$

with $F^{\mu}=F^{\mu \alpha} j_{\alpha}$ being the force-density 4-vector which describes the back action of the fields produced by the charged particles on the same particles.

From the differential conservation law $\partial_{\nu} \widetilde{T}^{\mu \nu}=0$, the integral law of conservation results as

$$
\int \mathrm{d} \vec{r} T^{\mu 0}=P^{\mu}=\text { const }
$$

where $P^{\mu}$ is an energy-momentum 4 -vector. It should be noted here that the quantity

$$
\int d \vec{r} \theta^{\mu 0} \equiv \tau^{\mu 0}
$$


is not a 4 -vector. This means that it is impossible in principle to define correctly the notions of energy and momentum of both the particle free of its own Coulomb and vortex fields and the fields being produced by the particle and separated from it. It is only the particle being thought of as an elementary excitation of the charged matter together with the fields included in its definition that is a well-defined physical object.

The quantum theory of a self-acting electron given above differs qualitatevely from the theory of electromagnetic mass of an electron (the Abraham-Lorentz model). In the latter the stability of the electron is achieved by introducing special attractive forces being produced by a hypothetical matter field and compensating for the Coulomb repulsive forces. In the present theory, contrastingly, there are no additional forces and no material sources creating them. It is the electrically charged matter that is the only source of both the Coulomb forces and ones compensating them and holding the particle stable. The stability of the electron is due to the Coulomb self-action and is achieved by the use of a functional space with the indefinite metric.

As can be seen from the model of the electron discussed above, the Coulomb field plays a leading part in formation of the electron being considered as a clot of the charged matter localized in some region of space. It is evident, besides, that the Coulomb selfaction described by the last term in the right-hand side of (28) cannot be considered as a small perturbation. Indeed, the behaviour of the electron wave function considerably depends on self-action: when self-action is lacking (i.e., at $A=0$ in (29)), the electron wave function is a plane wave, whereas at $A \neq 0$ the wave function describes a soliton $[10,13]$, the state of a particle localized in space.

\section{Conclusion}

The solutions to the nonlinear equations (10) and (26) in the absence of vortex electromagnetic field were obtained and investigated in detail in $[8-11,13]$. Relaying on these solutions, we can draw the following conclusions:

1. The self-acting electron is a soliton which can be in different quantum states characterized by internal energy, dimensions and geometric shape.

2. The self-acting electron has a discrete internal energy spectrum, the size and geometric shape of the particle depending upon the value of its internal energy.

3. The atom consists of one or several electronic solitons interacting with the nuclear soliton.

4. Discreteness of the internal energy spectrum of the nuclear soliton is responsible for appearance of a band structure in the energy spectrum of a hydrogen atom, Balmer's spectrum being one of energy bands.

5. Discreteness of the internal energy spectrum of the electron and existence of energy bands in the atom offer great possibilities of using quantum transitions between internal energy levels of a particle, including the levels inside bands, for controlling intra-electron processes and producing new materials, electronic devices and technologies. 
Note that a self-acting electron cannot decay into fragments under influence of a perturbation. The particle can merely go from one quantum state to the other with the result that its charge distribution in space may vary considerably in size and shape. The immediate task of theoretical research is to obtain and investigate the solutions of the fundamental equations corresponding to both stationary and nonstationary states of the self-acting electron and to evaluate the intensity of quantum transitions between the internal energy levels.

\section{References}

[1] Schrödinger E., Ann. der Physik, 1926, V.81, 109-139.

[2] Barut A.O., Kraus J., Found. of Physics, 1983, V.13, 189.

[3] Barut A.O., van Huele J.F., Phys.Rev. A, 1985, V.32, N 6, 3187-3195.

[4] Barut A.O., Physica Scripta, 1988, V.21, 18.

[5] Barut A.O., Dowling J.P., Phys.Rev. A, 1987, V.36, N 2, 649-654.

[6] Fushchych W., Zhdanov R., Physics Reports, 1989, V.172, N 4, 123-174.

[7] Fushchych W., Shtelen W., Basarab-Horwath P., A new conformal-invariant nonlinear spinor equation, Linköping University, Preprint LiTH-MAT-R-93-05, 1993.

[8] Oleinik V.P., Quantum Electronics, 1993, V.44, 51-58.

[9] Oleinik V.P., Ibid., 1993, V.45, 57-79.

[10] Oleinik V.P., Ibid., 1994, V.47, 41-52.

[11] Oleinik V.P., Quantum electrodynamics describing the internal structure of electron. Gaugeindependent and covariant theory, Universität Leipzig, NTZ, Prepr. 7/1992.

[12] Haken H., Advanced synergetics. Instability hierarchies of self-organizing systems and devices, Springer-Verlag, Berlin, Heidelberg, New York, Tokyo, 1983.

[13] Arepjev Ju.D., Buts A.Ju., Oleinik V.P., To the problem of internal structure of electrically charged particles. Spectra of internal energy and charge distribution for a free electron and hydrogen atom, Prepr. of the Inst. of Semiconductors of the Ukraine, 8-91, Kiev, 1991. 